Development of Ultrasound Tomography for Breast Imaging: Technical Assessment

N. Duric, P. Littrup, A. Babkin, D. Chambers, S. Azevedo, K. Arkady, R. Pevzner, M. Tokarev, E. Holsapple

October 14, 2004

Medical Physics 
This document was prepared as an account of work sponsored by an agency of the United States Government. Neither the United States Government nor the University of California nor any of their employees, makes any warranty, express or implied, or assumes any legal liability or responsibility for the accuracy, completeness, or usefulness of any information, apparatus, product, or process disclosed, or represents that its use would not infringe privately owned rights. Reference herein to any specific commercial product, process, or service by trade name, trademark, manufacturer, or otherwise, does not necessarily constitute or imply its endorsement, recommendation, or favoring by the United States Government or the University of California. The views and opinions of authors expressed herein do not necessarily state or reflect those of the United States Government or the University of California, and shall not be used for advertising or product endorsement purposes. 


\section{Development of Ultrasound Tomography for Breast Imaging: Technical Assessment}

Nebojsa Duric ${ }^{1}$, PhD, Peter Littrup ${ }^{1}$, MD, Alex Babkin ${ }^{2}, \mathrm{PhD}$, David Chambers ${ }^{3}$, PhD, Steve Azevedo $^{3}$, Kalinin, Arkady ${ }^{2,5,6}$, PhD, Roman Pevzner ${ }^{2,4}$, PhD, Mikhail Tokarev ${ }^{2.4}$, PhD, Earle Holsapple ${ }^{1}$, BA

${ }^{1}$ Karmanos Cancer Institute, 110 East Warren, Hudson-Weber Building, Suite 504, Detroit MI 48201

${ }^{2}$ Groupvelocity, LLC, Albuquerque NM 87131

${ }^{3}$ Lawrence Livermore National Laboratory, Livermore CA 94551

${ }^{4}$ DECO Geophysical Ltd, Moscow Russia

${ }^{5}$ Moscow State University, Moscow Russia

${ }^{6}$ Deceased

Corresponding author: Nebojsa Duric, Phone: (313) 966 8862, FAX (313) 966 7322, email: duric@karmanos.org 


\begin{abstract}
Ultrasound imaging is widely used in medicine because of its benign characteristics and real-time capabilities. Physics theory suggests that the application of tomographic techniques may allow ultrasound imaging to reach its full potential as a diagnostic tool allowing it to compete with other tomographic modalities such as X-ray CT and MRI. This paper describes the construction and use of a prototype tomographic scanner and reports on the feasibility of implementing tomographic theory in practice and the potential of US tomography in diagnostic imaging.

Data were collected with the prototype by scanning two types of phantoms and a cadaveric breast. A specialized suite of algorithms was developed and utilized to construct images of reflectivity and sound speed from the phantom data. The basic results can be summarized as follows.

(i) A fast, clinically relevant US tomography scanner can be built using existing technology,

(ii) The spatial resolution, deduced from images of reflectivity, is $0.4 \mathrm{~mm}$. The demonstrated $10 \mathrm{~cm}$ depth-of-field is superior to that of conventional ultrasound and the image contrast is improved through the reduction of speckle noise and overall lowering of the noise floor.

(iii) Images of acoustic properties such as sound speed suggest that it is possible to measure variations in the sound speed of $5 \mathrm{~m} / \mathrm{s}$. An apparent correlation with $\mathrm{X}$-ray attenuation suggests that the sound speed can be used to discriminate between various types of soft tissue.

(iv) Ultrasound tomography has the potential to improve diagnostic imaging in relation to breast cancer detection.
\end{abstract}

Key words: breast cancer, ultrasound tomography, diagnostic imaging, tissue characterization 


\section{Introduction}

Ultrasound (US) provides a safe, non-invasive, non-ionizing imaging modality for a wide spectrum of medical imaging needs. It is commonly used in fetal, cardiac, orthopedic, abdominal, genitourinal and breast imaging. Recent studies have demonstrated the effectiveness of US imaging in detecting breast cancer ${ }^{1}$, particularly for women with dense breasts. At the same time, other studies have raised questions about the efficacy of mammography ${ }^{2}$. However, despite these developments, US is used only as an adjunct to X-ray mammography. A major reason for ultrasound's adjunctive role is its limited imaging capabilities. Specifically, the operator dependence, limited resolution and contrast, the presence of speckle noise and artifacts are key factors which have prevented US imaging from direct comparison, let alone competing with X-ray mammography, CT and MRI.

Efforts to improve diagnostic accuracy of current US may be best represented by the success of the 'Stavros/Colorado' criterion for mass margin evaluation ${ }^{3}$. The $98 \%$ accuracy obtained for defining a benign mass (criteria of a well-defined, ovoid mass) was sufficient for ATL, Inc. to obtain separate Food and Drug Administration approval for characterizing benign breast masses with their high-resolution transducer in $19966^{4}$. Further advances have led to compound imaging ${ }^{5}$ (SonoCT, ATL/Phillips) that produces a single averaged image from 3-9 single angled scans. This advancement has resulted in better mass margin identification by reduction, of speckle, clutter and ultrasound artifacts ${ }^{6}$.

These developments suggest that a major evolutionary trend in US imaging technology is toward tomographic devices. However, none of the current multi-view devices, such as SonoCT, are truly tomographic, in the sense of X-ray CT and MRI, in that they operate over a limited range of viewing angles and do not make use of the transmitted energy. True tomographic techniques have the potential to measure and interpret the scattered field, produced by the interaction of the US pulse with the tissue through which it is propagating, thereby markedly expanding US imaging potential. Although the computational and data acquisition requirements have been major barriers to successful implementation of US tomography, recent technological advances in computing and electronics have led to renewed optimism for the development of clinically relevant tomography-based US scanners. A number of investigators and their groups are actively developing tomography-based US scanners ${ }^{7-18}$. After the pioneering work of Greenleaf ${ }^{8}$ in tissue characterization and more recently work in US tomography by Andre et $\mathrm{al}^{7}$, current work is being carried out on a number of fronts and includes Johnson et $\mathrm{al}^{9}$ (TechniScan Inc), Marmarelis' group at USC ${ }^{10}$, Waag's group at Rochester ${ }^{11}$ and the work at the Karmanos Cancer Institute (KCI) in Detroit ${ }^{12-18}$.

In this paper we describe the results of US tomography research at the KCI. In particular, we focus on the experimental results arising from the use of a prototype US scanner developed in conjunction with the Lawrence Livermore national Laboratory (LLNL). In section II we describe the basic physical principles of US tomography. The general methods associated with the data collection and analysis are described in section III. Section IV describes the imaging experiments undertaken with the prototype scanner. The clinical relevance of the results is discussed in 
section V. A summary of the work and the future trajectory of this research is presented in section VI.

\section{Physical Principles of US Tomography}

\section{A. The Scattered Field}

When an ultrasound pulse propagates through tissue, its path is governed by the variations in tissue properties. The sensitivity of the US pulse to these tissue variations leads to the formation of a complex scattered field (Fig 1). The scattered field therefore contains information about the propagation medium, in this case, human tissue. We now discuss the theoretical principles that define the interaction of US with tissue and lead to the formation of a scattered field.

\section{Reflection and refraction}

When an ultrasound wave passes through an interface between two materials at an oblique angle, and the materials have different indices of refraction, it produces both reflected and refracted waves. Refraction takes place at an interface due to the different velocities of the acoustic waves within the two materials. The velocity of sound in each material is determined by the material properties (elastic modulus and density) for that material. Snell's law equates the ratio of material velocities $V_{1}$ and $V_{2}$ to the ratio of the sines of incident $\left(\theta_{1}\right)$ and refraction $\left(\theta_{2}\right)$ angles, as shown in Fig 2. Note that in the diagram, there is a reflected longitudinal wave ( $\left.\mathrm{V}^{\prime}{ }_{1}\right)$ shown. This wave is reflected at the same angle (relative to normal) as the incident wave because the two waves are traveling in the same material and, therefore, have the same velocities. The strength of the reflected wave is determined by the acoustic impedances of the two media.

The acoustic impedance $(\mathbf{Z})$ of a material is defined as the product of density $(\rho)$ and acoustic velocity $(\mathbf{V})$ of that material.

$$
Z=\rho V
$$

In the case of normal incidence, the reflected energy is related to the change in acoustic impedance, as follows

$$
R=\left[\frac{Z_{2}-Z_{1}}{Z_{2}+Z_{1}}\right]^{2}
$$

It should be noted that the sum of the transmitted sound energy plus the reflected sound energy is a conserved quantity. In the absence of absorption, no energy is lost directly as a result of these interactions. 
The reflections and refractions occur because of variations in the acoustic impedance of tissue. Consequently, when an US pulse is injected into tissue the output is not a simple transmission or back-reflection of the pulse but rather an extended acoustic field, the so-called "scattered field". The scattered field contains information about the reflecting boundaries of the tissue as well as acoustic properties of the medium as described below.

\section{Sound Speed}

The speed of sound within a material is a function of the properties of the material and is independent of the amplitude of the sound wave and nearly independent of the acoustic frequency. The general relationship between the speed of sound in material and its density and elastic constants is given by the following equation:

$$
\mathbf{V}=\sqrt{\frac{c}{\rho}}
$$

where $\mathbf{V}$ is the speed of sound, $\mathbf{c}$ is the elastic constant, and $\rho$ is the material density. Variations in sound speed contribute to the reflections and refractions discussed above. The transmitted component of the scattered field contains information about the velocity variations in the propagating medium.

\section{Attenuation}

The combination of absorption and scattering is referred to as attenuation which is a measure of how much energy is lost from a straight path connecting two points. Tissue is characterized by an inhomogeneous distribution of sound speed and density, making an ultrasound pulse experience multiple refractions and reflections as it propagates through tissue. Furthermore, diffraction occurs when ultrasound waves interact with structures in the tissue that are comparable in size to the wavelength of the ultrasound. The combination of multiple refractions, reflections and diffraction leads to scattering. Scattering causes ultrasound energy to be redirected in directions away from the incident direction. This energy is not lost but simply redirected. Absorption is defined as the conversion of ultrasound energy into heat during the propagation of the ultrasound pulse. In this case, ultrasound energy is lost and cannot be recovered.

Attenuation is defined mathematically as the decay rate of the US pulse amplitude as the pulse propagates through the material. The amplitude of a spatially decaying plane wave can be expressed as:

$$
A=A_{0} e^{-\alpha(f) z}
$$


In this expression $A_{0}$ is the amplitude of the propagating wave at a reference location. The amplitude $A$ is the reduced amplitude after the wave has traveled a distance $z$ from that initial location. The quantity $\alpha$ is the attenuation coefficient of the wave traveling in the $z$-direction. For human tissue the bulk average attenuation coefficient is a strong function of frequency, $f$, and can be expressed as

$$
\alpha=0.5 \mathrm{~dB} / \mathrm{MHz} / \mathrm{cm} \text {. }
$$

In practice, the attenuation coefficient varies relative to its mean value because of variations in tissue type. The scattered field contains information on the attenuation of US signals because the detected pulse amplitude can be compared with the original (transmitted) pulse amplitude and the level of attenuation inferred.

As can be seen from the above discussion, the scattered field is shaped by the propagation of US in an inhomogeneous medium that is governed by reflection, refraction, and attenuation and contains information about the variations of acoustic properties of the medium. In order to use measurements of the scattered field to determine the acoustic properties of the insonofied volume it is necessary to solve the "inverse" problem, that is, to determine unknown causes based on observation of their effects. Within tissue the causes are reflection, refraction and attenuation of US pulses and the effects are the spatial and time-dependent properties of the scattered field. In general, such problems cannot be solved analytically and numerical algorithms are needed to perform the inversion. Such algorithms are described in section III.

\section{B. Conventional US imaging}

Conventional US is driven by the need for real-time data acquisition and display. Since computational speed is the key to real-time functionality, some of the complex physics associated with US propagation is traded off. Three of the major trade-offs involve the following simplifications.

a. Modeling the propagation of US using straight-ray theory. Straight-ray theory is only a basic approximation of the true physics of acoustic wave propagation and is only valid for purely homogenous media.

b. Assuming a 2D geometry and collecting only the directly backscattered reflections. The arrival of the reflected signals is recorded only back along the direction of the emitted pulses (i.e. only the so called 180 degree reflections are recorded). In reality, the emitted pulses interact so strongly with the tissue that a "scattered field" of acoustic energy is produced (Fig. 2). Acoustic energy is distributed in all directions so that only a small fraction of the US energy comes directly back to the detectors. Consequently, the strength of the return signal is weak and it is necessary to "beam form" (focus energy in a specific direction) in order to amplify the return signal (with most of the emitted energy still lost). 
c. Operator dependence. Real-time imaging can be implemented for modest volumes of tissue but requires manual translation of the device in order to image the entire volume of interest.

These trade-offs for real time imaging have compromised image quality and resulted in a loss of diagnostic information contained in the US-tissue interaction. A tomographic approach has the potential to "undo" these trade-offs leading to a marked increase in the signal-to-noise ratio while reducing artifacts and yielding higher quality images for greater clinical "sensitivity". Furthermore, the US signals that propagate through the breast, and which are never reflected back, contain additional information. These transmitted signals can be used to calculate acoustic parameters, not contained in the reflection data, such as sound speed and attenuation, possibly leading to greater clinical "specificity",7-26.

\section{Implementation of US Tomography}

Tomographic principles have been implemented in a working US scanner. As described in the next section, an early prototype was designed and built in order to carry out phantom experiments that were aimed at:

(i) defining an engineering solution for a human-use clinical scanner,

(ii) developing the associated algorithms for image reconstruction,

(iii) assessing the potential of the clinical scanner in diagnostic imaging of the breast.

Major design goals included the ability to penetrate tissue with minimal attenuation and to generate images with good spatial resolution and contrast even at the center of a highly attenuating cadaveric breast.

Although the resolution, $\theta$, of tomographic images is governed generally by the acoustic properties of the medium, the wavelength, $\lambda$, of the ultrasound may be a limiting factor. This limitation can be estimated using the following simple considerations. In the case of reflection imaging,

$$
\theta=0.5 \lambda \text {. }
$$

The relationship between wavelength and frequency is given by

$$
\lambda=\frac{\mathrm{v}}{f}
$$

where $V$ is the speed of sound in tissue and $f$ is the ultrasound frequency. Combining equations (6) and (7) yields

$$
\theta=0.5 \frac{\mathrm{v}}{f}
$$


For a target resolution of $0.5 \mathrm{~mm}$, inversion of the above equation yields $f=1.5 \mathrm{MHz}$. Examination of equation 5 shows that this choice of frequency leads to a reduction in the attenuation coefficient of $6 \mathrm{~dB}$ relative to a standard B-scan device operating at $9 \mathrm{MHz}$. Therefore, the choice of $1.5 \mathrm{MHz}$ is optimal for our purposes because it leads to a reduction in attenuation while maintaining high resolution. The reduction in attenuation has the added advantage of allowing the introduction of simplifying assumptions in the reconstruction algorithms. One such assumption is the use of a 2.5-D tomographic approach in which data are acquired in 2-D planes which are reconstructed into 2-D images and stacked to reconstruct a 3-D volume. This approach assumes that little scattering takes place out of the plane so that 2-D algorithms can be used to produce the tomographic images. Consequently, the choice of the relatively low US frequency of $1.5 \mathrm{MHz}$ enables this simpler approach and justifies avoiding the full 3-D approach.

\section{General Methods}

\section{A. Experimental Tomographic Scanner}

KCI has collaborated with Lawrence Livermore National Laboratory (LLNL) to build a proof-ofconcept engineering prototype to test the feasibility of building a clinically relevant device with available technology. The resulting scanner utilized an independently revolving pair of transducers to emulate a high density array of ultrasonic transducers. A schematic of the scanner is shown in Fig. 3.

After successful testing and debugging, the prototype was used to carry out a series of experiments. The experimental setup is shown in Fig. 4. Two transducers are mounted on the scanner. They are identical line transducers, $0.38 \mathrm{~mm}$ wide and $12 \mathrm{~mm}$ high. The relative positions of the two transducers are encoded to better than $0.05 \mathrm{~mm}$. For each position of the transducer, the receiver is moved around a 320 degree circular path of radius $15 \mathrm{~cm}$, (the 40 degree gap was the result of mechanical limitation of the two rotation stages used in the experimental setup). The transmitter emits a pulse for each position of the receiver for up to 1600 receiver positions (position increments of $0.2 \mathrm{deg}$ ). The transmitter position is also moved along the circular path and the firing sequence repeated for each new position of the transmitter. The transmitter is moved in increments of 1 degree for a total of 360 positions. The overall firing sequence is illustrated in Fig. 5. The data presented here were generated by using a total of 320 receiver positions and 360 transmitter positions, for a total of over $10^{5}$ receive-transmit pairs. The targets were placed at the center of the ring with the long axis of the cylindrical phantom oriented vertically relative to the plane of the ring. Each data set represents a 2-D slice through the target. The ring plane was translated in the vertical direction allowing for 3-D reconstructions from stacked 2-D planes of data. All scans were performed at $10 \mathrm{~mm}$ slice thickness, as determined by the beam width of the transducers.

The data sets resulting from the above scans were typically 2 GB per slice in size and were stored on a raid-type disk farm (Fig 3). The raw data sets consisted of measured pulse trains, processed to determine the frequency spectrum and arrival time for each pulse. The arrival times were used to construct reflection and sound speed images while the pulse spectrum data were used to determine the attenuation. 


\section{B. The Phantoms}

Experiments were designed to image progressively more complex targets in order to test the basic capabilities of the scanner. Two basic phantoms were used to ascertain punctate resolution and mass margins (i.e. microcalcifications and cysts). Each is a cylindrically shaped phantom 40 $\mathrm{mm}$ in diameter by $\sim 150 \mathrm{~mm}$ in height and filled with Agar, $\mathrm{N}$-propanol, and $\mathrm{H}_{2} \mathrm{O}$ proportioned to attain an internal sound speed of $\sim 1.54 \mathrm{~mm} / \mu \mathrm{s}$. The phantoms are housed within a Latex casing. The "microcalcification" phantom has 5 pairs of nylon wires ( $0.2 \mathrm{~mm}$ diameter) running the length of the phantom with pair spacings from 2 to $5 \mathrm{~mm}$. The pairs form a pentagon whose vertices are $\sim 12.5 \mathrm{~mm}$ from the center of the phantom. A cross-sectional slice of this phantom represents "microcalcifications" of diameter $0.2 \mathrm{~mm}$ (Fig. 6). The "cyst" phantom has 2 inclusions (10 mm diameter latex tubes) running the length of the phantom and filled with a water/alcohol mixture having a lower sound speed relative to the surrounding medium. The inclusions in the cyst phantom mimic the slower sound speed in fluid filled cysts in a low contrast setting. Furthermore, naturally occurring deformations in the inclusions helped mimic invagination and exophytic features. A cross-sectional slice of the cyst phantom represents a pair of “cysts” each having a diameter of $1 \mathrm{~cm}$ (Fig. 6). A third target used in this study is a normal cadaveric human breast (Fig. 6) placed in formalin and sealed in a $100 \mathrm{~mm}$ diameter, cylindrical container with fiducial nylon wires running the length of the outside of the container at four locations (quadrants). The purpose of this phantom is to test the ability of the scanner to image the complex, highly scattering medium of the breast.

The phantoms were placed in the water tank with their long axes oriented vertically, such that the scanning process produced cross sectional slices at one or more levels through the test object.

\section{Image Reconstruction}

Generally, a tomographic reconstruction is always based on a method that "blurs" over space some characteristic property of the medium that is responsible for the overall "integral" effect. For example, an increase in the propagation time of a sound wave that is caused by a variation of the sound speed in the medium can be approximated by assuming a distribution of unit cells of a finite size where the sound speed is assumed constant within a cell. The way this is done and the manner in which the reconstructions are implemented into practical algorithms are now described.

The reconstruction algorithms are grouped into two basic types, according to whether they use the recorded back-scattered field or the forward-scattered field to construct the images. The former are referred to as reflection algorithms while the latter are termed transmission algorithms. The relevant geometries are shown schematically in Fig. 7. Early results from these algorithms have been described ${ }^{7,8}$.

\section{Reflection Algorithms}


The scattered field contains information about the reflecting boundaries located within the insonofied volume. The reflective boundaries are defined by changes in acoustic impedance in the propagating material. We have developed algorithms that analyze the scattered field and determine the locations of the reflective boundaries. The output of such algorithms is a 2-d image of the reflective surfaces. The results shown here are based on a class of algorithms known as migration algorithms. They are based on a fundamental principle that was first formulated by Claerbout ${ }^{27}$ as "reflectors exist at points [in the medium] where the first arrival of the downgoing wave is time-coincident with the upgoing wave".

For practical realization of this method it is important to correctly determine the propagation times of (i) the transmitted acoustic wave that travels from a sound transducer to some reflecting boundary within the investigated medium and (ii) the reflected wave generated at this boundary that travels to the sound receiver. The most important parameter of the medium that has to be known in order to complete the migration transformation is the speed of the ultrasound pulse. In the case of a sound wave propagating through the breast tissue all the variations of the sound speed are relatively small and the average speed of migration can be safely assumed constant. This assumption also allows us to stay within the "straight ray" approximation meaning that all the refraction effects are generally small and can be neglected. The spatial resolution of this reconstruction is generally governed by the wavelength of the ultrasound and can be estimated based on the simple considerations described in the Section II(C).

In our experimental setup, measurements of the received pulse train exist for a fixed set of angles for all positions of the sound transmitter (all views). In principle, this fixed set of angles could correspond to the entire circular aperture (i.e. 360 degrees). In practice, a narrower range of angles (typically 40 degrees) was used in order to speed up the reconstruction process. Each of the recorded pulse trains allows one to construct a position on the reflecting boundary. By using a large number of pulse train measurements we are able to improve on the accuracy of locating and defining the reflecting boundary and its diffracting properties, relative to conventional ultrasound, leading to high-resolution high-dynamic range reflection images.

\section{Transmission Algorithms}

This class of algorithms is based on the same physical principles as conventional Computer Tomography (CT) but adopted specifically for ultrasound implementation. The main difficulty in implementation of the ultrasound tomography is that in this case the data set is almost always incomplete and standard methods used in CT (like the inverse Radon transformation for example) cannot be directly applied. Instead, to reconstruct the acoustic properties of the medium (and more importantly the spatial distribution of the acoustic properties) more complex and computationally intense methods have to be utilized.

The basic idea of the ultrasound tomographic transformation can be formulated as follows. Let's assume that we have $\mathrm{N}$ transmitter/receiver pairs (and correspondingly the same number of rays that cross the investigated media). Generally, the reconstruction is done using a rectangular grid and within each of the unit cells we assume the sound speed/attenuation to be constant. Let the total number of unit cells be equal to $\mathrm{M}$. 
The sound travel time along the ray $i$ is $T_{i}=\sum_{j=1}^{M} l_{i, j} b_{j}$, where $l_{i, j}$ is the length of this ray's path inside the cell $j$ ( if the ray doesn't cross this cell this value is zero) and $b_{\mathrm{j}}$ is the reverse sound speed in this cell. This means that the distribution of sound speed can be reconstructed by solving the system of $\mathrm{N}$ equations $\left\{T_{i}=\sum_{j=1}^{M} l_{i, j} b_{j}\right.$. In practice the solution of this system of equations is difficult to obtain since the values of $\mathrm{T}_{\mathrm{i}}$ are large and $l_{i, j}$ are small. For this we introduce an initial model $\left\{b_{j}^{0}\right\}$. For this model we have $\left\{T_{i}^{0}=\sum_{j=1}^{M} l_{i, j} b_{j}^{0}\right.$. By subtracting the second system from the first one, we arrive at $\left\{T_{i}-T_{i}^{0}=\sum_{j=1}^{M} l_{i, j}\left(b_{j}-b_{j}^{0}\right)\right.$, or $\left\{\Delta T_{i}=\sum_{j=1}^{M} l_{i, j} \Delta b_{j}\right.$. It is not uncommon that the number of equations in this system exceeds the number of unknowns. For this reason the solution has to be found in the "least squares sense" taking special care to ensure the stability of the solution.

Since the actual value of $\mathrm{N}$ is generally very large (around 250,000 or even larger) only the rays that actually cross the investigated object have to be used in the tomographic reconstruction. In addition, the implication of some additional selection rules, for example selecting the cells inside the object that have been crossed by the rays no less then a certain number of times, allows the algorithm to operate with a significantly reduced system of equations $(10,000$ to 30,000$)$ without any noticeable degradation of the resultant reconstructed image.

\section{Imaging Experiments}

The two phantoms and cadaveric breast specimen were scanned by the prototype at a frequency of $1.5 \mathrm{MHz}$, a clinical reflection ultrasound unit (GE Logiq 600, using a broadband 6-11 MHz linear transducer array) and a clinical CT scanner (GE Lightspeed Quad detector array). All scans were performed at 10 millimeter slice thickness to generate multiple tomographic images per phantom. Each slice required a two-hour scan time to emulate a fully populated array within the prototype. The X-ray CT scans were performed at $1.25 \mathrm{~mm}$ slice thickness.

Images were reconstructed using the reflection and transmission algorithms described above. Images of reflectivity and sound speed are shown in Fig. 8, 9 and 10 for the three phantoms scanned in this study. The image quality for the various phantoms is summarized in the performance matrix below. The terms in the matrix are described as follows.

Background: All phantoms were immersed in water. This water is referred to as the background.

Interior: The interior of each phantom is made of agar, a material whose sound speed mimics that of breast tissue. Its typical value is $1540 \mathrm{~m} / \mathrm{s}$ though there are unknown variations from phantom to phantom. The number entered in this column is the measured sound speed for the interior. 
Microcalcifications: The microcalcification phantom contains $0.2 \mathrm{~mm}$ diameter threads aligned vertically along the axis of the agar cylinder. In cross-section these threads appear as circular inclusions, well suited for measuring the resolution. However, their small size prevents accurate sound speed measurements in their vicinity. The diffraction of the ultrasound waves around these wires results in higher sound speeds near the wire. However, these sound speeds are not representative of the wire material itself.

Cysts: The small imbedded cylinders contain a water-alcohol mixture with a sound speed lower than that of the surrounding agar. These cylinders are meant to mimic water-filled cysts and they test the algorithm's ability to measure sound speeds in a low-contrast setting. The values entered in this column are the average sound speeds in the interiors of these "cysts".

Resolution: The ability to measure fine structure in an image. The resolution was estimated by examining the images of the microcalcification and cyst phantoms. Knowing the size of the objects embedded in the phantoms has allowed us to estimate the resolution of the prototype. In the case of the microcalcification phantom this was done by comparing the apparent diameters of the wires with the known values. In case of the cyst phantom it was done by measuring the thicknesses of the cysts walls. In both cases, the following formula was used

$$
D_{\text {apparent }}=\sqrt{D_{\text {true }}^{2}+D^{2}{ }_{p s f}}
$$

to solve for the diameter of the point spread function $\left(\mathrm{D}_{\mathrm{psf}}\right)$, where the $*$ symbol represents convolution and the profiles were assumed to be Gaussian.

Accuracy: The ability to differentiate variations in sound speed as a function of location within the breast is an important aspect of tissue characterization. Figures in this column refer to the minimum percentage variations that can be unambiguously discerned relative to water. In the case of reflection the accuracy refers to the lowest amplitude pulse that is discernible on reflection, relative to the strongest emitted pulse. The lower the number the better. 
Breast Tissue

\begin{tabular}{|lllll|}
\hline & Background & Interior & Resolution & Accuracy \\
Reflection & & & $0.5 \mathrm{~mm}$ & $0.1 \%$ \\
$\begin{array}{l}\text { Sound } \\
\text { Speed }\end{array}$ & $1485 \pm 5 \mathrm{~m} / \mathrm{s}$ & $1425 \pm 5$ to $1475 \pm 5 \mathrm{~m} / \mathrm{s}$ & $0.4 \mathrm{~mm}$ & $0.3 \%$ \\
\hline
\end{tabular}

\section{Cyst Phantom}

\begin{tabular}{|llllll|}
\hline & Background & Interior & Cysts & Resolution & Accuracy \\
Reflection & & & & $0.4 \mathrm{~mm}$ & $0.1 \%$ \\
$\begin{array}{l}\text { Sound } \\
\text { Speed }\end{array}$ & $1485+/-3 \mathrm{~m} / \mathrm{s}$ & $1535+/-5 \mathrm{~m} / \mathrm{s}$ & $1510+/-3 \mathrm{~m} / \mathrm{s}$ & $1.0 \mathrm{~mm}$ & $0.3 \%$ \\
\hline
\end{tabular}

Microcalcification Phantom

\begin{tabular}{|llllll|}
\hline & Background & Interior & Points & Resolution & Contrast \\
Reflection & & & & $0.3 \mathrm{~mm}$ & $0.1 \%$ \\
$\begin{array}{l}\text { Sound } \\
\text { Speed }\end{array}$ & $1485+/-3 \mathrm{~m} / \mathrm{s}$ & $1545+/-5 \mathrm{~m} / \mathrm{s}$ & $1610+/-5 \mathrm{~m} / \mathrm{s}$ & $1.0 \mathrm{~mm}$ & $0.3 \%$ \\
\hline
\end{tabular}




\section{Discussion}

The engineering prototype was used to gain insight on the feasibility of implementing a clinical scanner. A key step in that process is the analysis of the data acquired with the engineering prototype. The most relevant data set used in this study was from the scans of the cadaveric breast. Images based on the acoustic properties of reflectivity and sound speed can be compared with images made from X-ray CT scans. The latter were used to establish a baseline reference (the "truth") in order to help gauge the imaging quality of the US reconstructions.

Fig. 11 shows a US reflection image of the cadaveric breast at $1.5 \mathrm{MHz}$ and an X-ray CT image, for comparison. The cadaveric breast specimen shows predominantly fatty tissue with thin but prominent internal fibrous bands. The US image shows the overall specimen configuration with excellent morphologic comparison to the CT scan. There is also no loss of signal or resolution seen in the near or far field. Despite the low frequency of the US tomography image, the fine architectural detail of internal fibrous bands is similar to that in the CT image. The correlation between reflectivity of the fibrous bands and their X-ray attenuation suggests that US reflectivity may correlate with X-ray absorption (which is proportional to tissue density). Thus is not too surprising since, according to equation 2, the reflectivity is related to changes in acoustic impedance, which in turn, are related to changes in tissue density.

The reflection data provide high-resolution images of reflective boundaries but relatively little quantitative information. As noted earlier quantitative information can be added through the calculation of acoustic properties such as sound speed. Fig. 12 shows the reflection image with the sound speed information superimposed. The resulting image can be thought of as a reconstruction that utilizes both the reflected and transmitted components of the scattered US field. The most interesting aspect of the combined image is the ability to differentiate various tissue types. The labels in Fig. 12 identify four distinctly different regions. On the basis of their $\mathrm{X}$-ray morphology and attenuation properties these have been qualitatively identified as fibrous structure, fatty tissue, formalin fluid and high density tissue. The fibrous bands, evident in the Xray image as filamentary structures, are also evident in the US image. The fibrous structure seen in the US image is revealed best by the acoustic property of reflectivity as evidenced by its presence in the reflection image (Fig. 11). The fatty tissue is characterized in the CT images by regions of smooth, low-X-ray-absorption (low density) regions of tissue. In the US image this same region is characterized by a smooth distribution of slowly varying sound speed. In both cases, the fatty tissue appears as an underlying substrate within which the fibrous bands are embedded. The region labeled in Fig. 12 as high-density tissue refers to the strips of fibrous tissue that have apparently high absorption to X-rays. Although we do not have the histological study to determine its nature, we do note that it has high sound speed relative to the rest of the breast. In fact, it has the highest sound speed values of the four regions identified in Fig. 12. In the case of the fourth region, the formalin fluid, it is identified in the X-ray image as a smooth, feature-free region having X-ray absorption levels intermediate to those of the fatty tissue and high-density tissue. Interestingly, in the US image, the formalin liquid has a sound speed intermediate between the fatty tissue and high-density tissue. 
Comparison of the US image with the "truth image" suggests that it is possible to identify four distinct regions on the basis of acoustic properties alone. The regions and the acoustic property by which they are identified are listed below.

(i) Fibrous bands: reflectivity

(ii) Fatty tissue: sound speed: $1425 \mathrm{~m} / \mathrm{s}$

(iii) Dense tissue: sound speed: $1525 \mathrm{~m} / \mathrm{s}$

(iv) Formalin fluid: sound speed: $1475 \mathrm{~m} / \mathrm{s}$

These results suggest that US tomography has the potential to characterize tissue in addition to providing high resolution morphological information. The potential for clinically relevant tissue characterization and the promise of identifying cancer on the basis of acoustic properties will be explored in a future paper. 


\section{Conclusions and Future Work}

A prototype US tomographic scanner was built for the purpose of utilizing tomographic techniques in support of developing improved US diagnostic imaging. Using a pair of transducers on independent rotation stages the scanner simulated a ring of up to 360 transmit transducers and up to 1600 receivers. The prototype was used to scan a variety of phantoms and cadaveric tissue. Images of acoustic properties were successfully constructed from the scanned data. The phantom studies demonstrate the following capabilities

a. The spatial resolution, deduced from images of reflectivity, is $0.4 \mathrm{~mm}$. The demonstrated $10 \mathrm{~cm}$ depth-of-field is superior to that of conventional ultrasound and the image contrast is improved through the reduction of speckle noise and overall lowering of the noise floor.

b. Images of acoustic properties such as sound speed suggest that it is possible to measure variations in the sound speed of $5 \mathrm{~m} / \mathrm{s}$. An apparent correlation with Xray attenuation suggests that the sound speed can be used to discriminate between various types of tissue.

c. A fast, clinically relevant US tomography scanner can be built using existing technology.

d. Ultrasound tomography has the potential for improved diagnostic imaging in relation to breast cancer detection.

Although this early prototype was too slow to be used in clinical studies it did demonstrate a level of imaging capability that would be competitive with existing imaging modalities if implemented in a clinical scanner. The next step is to translate this imaging capability into a faster, clinically-relevant device. To that end we are developing a clinical prototype capable of in-vivo human imaging. The clinical prototype and the associated clinical studies are the subject of a future paper. 


\section{Acknowledgements}

The authors thank the staff of Techniscan Inc for their contributions and advice relating to the computational algorithms. We also thank and recognize many useful discussions with Michael Andre, PhD (UC San Diego), James Greenleaf, PhD (Mayo Clinic) and Frank Wuebbeling (U Muenster). The authors would also like to thank Richard Leach, Jeff Mast, Jim Candy, and the NDE group at Lawrence Livermore National Laboratory for help in acquiring the data and initial analysis. A portion of this work was performed under the auspices of the U. S. Department of Energy by the University of California, Lawrence Livermore National Laboratory under Contract No. W-7405-Eng-48

\section{References}

1. Kolb, T.M., Lichy, J. and Newhouse, J.H. Comparison of the Performance of Screening Mammography, Physical Examination, and Breast US and Evaluation of Factors that Influence Them: An Analysis of 27,825 Patient Evaluation. Radiology, 2002; 225: 165 - 175

2. Gotzsche PC, Olsen O. Is screening for breast cancer with mammography justifiable? Lancet 2000; 355:129-134.

3. Stavros AT, Thickman D, Rapp CL, Dennis MA, Parker SH, Sisney GA. Solid breast nodules: use of sonography to distinguish between benign and malignant lesions. Radiology. 1995;196:123-34.

4. Federal Register 60712-60713 (1996). (http://www.rsna.org/REG/research/regulatory/wfprfcexamples.htmlT)

5. Entrekin RR, Porter BA, Sillesen HH, Wong AD, Cooperberg PL, Fix CH. Real-time spatial compound imaging application to breast, vascular, and musculoskeletal ultrasound.Semin Ultrasound CT MR 2001 Feb;22(1):50-64

6. Shapiro RS, Simpson WL, Rausch DL, Yeh HC. Compound spatial sonography of the thyroid gland: evaluation of freedom from artifacts and of nodule conspicuity. AJR Am J Roentgenol 2001 Nov;177(5):1195-8

7. J. F. Greenleaf, A. Johnson, R. C. Bahn, B. Rajagopalan:Quantitative cross-sectional imaging of ultrasound parameters. 1977 Ultrasonics Symposium Proc., IEEE Cat. \# 77CH1264-1SU, pp. 989- 995,1977.

8. M. P. Andre, H.S. Janee, P. J. Martin, G. P. Otto, B. A. Spivey, D. A. Palmer, "High-speed data acquisition in a diffraction tomography system employing large-scale toroidal arrays," International Journal of Imaging Systems and Technology 8, pp.137-147, 1997.

9. Johnson S. A., Borup, D. T., Wiskin J. W., Natterer F., Wuebbling F., Zhang Y., Olsen C. Apparatus and Method for Imaging with Wavefields using Inverse Scattering Techniques. United States Patent 6,005,916 (1999).

10. Marmarelis, V.Z., Kim, T., Shehada, R.E. Proceedings of the SPIE: Medical Imaging 2003; San Diego, California; Feb. 23-28, 2002. Ultrasonic Imaging and Signal Processing - Paper 5035-6.

11. Liu, D.-L., and Waag, R. C. "Propagation and backpropagation for ultrasonic wavefront design," IEEE Trans. on Ultras. Ferro. and Freq. Contr. 44(1):1-13 (1997).

12. Littrup PJ, Duric N, Azevedo S, Chambers DH, Candy JV, Johnson S, Auner G, Rather J and Holsapple ET. Computerized ultrasound risk evaluation (CURE) system: Development of combined transmission and reflection ultrasound with new reconstruction algorithms for 
breast imaging. Proceedings of the $26^{\text {th }}$ International Acoustical Imaging Symposium; Windsor, Canada; Sept. 9-12, 2001.

13. Littrup PJ, Duric N, Leach Jr. RR, Azevedo SG, Candy JV, Moore T, Chambers DH, Mast JE and Holsapple ET. Characterizing tissue with acoustic parameters derived from ultrasound data. Proceedings of the SPIE: Medical Imaging 2002; San Diego, California; Feb. 23-28, 2002. Ultrasonic Imaging and Signal Processing - Paper 4687-43.

14. Leach Jr. RR, Azevedo SG, Berryman JG, Bertete-Aguirre HR, Chambers DH, Mast JE, Littrup PJ, Duric N, Wuebbeling F. A comparison of ultrasound tomography methods in circular geometry. Proceedings of the SPIE: Medical Imaging 2002; San Diego, California; Feb. 23-28, 2002. Ultrasonic Imaging and Signal Processing - Paper 4687-44.

15. Duric N, Littrup PJ, Leach Jr. RR, Azevedo SG, Candy JV, Moore T, Chambers DH, J. Mast JE and Holsapple ET. Using Data Fusion to Characterize Breast Tissue. Proceedings of the SPIE: Medical Imaging 2002; San Diego, California; Feb. 23-28, 2002. Ultrasonic Imaging and Signal Processing - Paper 4687-39.

16. Azevedo SG, Moore T, Huber RD, Ferguson W, Leach Jr. RR, Benson S, Duric N, Littrup PJ and Holsapple ET. Apparatus for circular tomographic ultrasound measurements. Proceedings of the SPIE: Medical Imaging 2002; San Diego, California; Feb. 23-28, 2002. Ultrasonic Imaging and Signal Processing - Paper 4687-12.

17. Duric N, Littrup P, Holsapple E, Babkin A, Duncan R, Kalinin A, Pevzner R and Tokarev M. Ultrasound Imaging of Breast Tissue. Proceedings of the SPIE: Medical Imaging 2003; San Diego, California; Feb. 21-26, 2003. Ultrasonic Imaging and Signal Processing - Paper 5035-4.

18. Huang LJ, Duric N, Littrup, P. Ultrasonic Breast Imaging Using a Wave-Equation Migration Method. Proceedings of the SPIE: Medical Imaging 2003; San Diego, California; Feb. 21-26, 2003. Ultrasonic Imaging and Signal Processing - Paper 5035-4.

19. Goss, S.A., Johnston, R.L. and Dunn, F. Comprehensive compilation of empirical ultrasonic properties of mammalian tissues. J Acoust Soc AM 1978; 64: 423-457

20. F. A. Duck: Physical properties of tissue. Academic Press, London, 1990

21. Edmonds, P.D., Mortensen, C.L., Hill, J.R., Holland, S.K., Jensen, J.F., Schattner, P. and Valdes, A.D. Ultrasound tissue characterization of breast biopsy specimens. Ultrasound Imaging 1991; 13:162-185.

22. Weiwad W, Heinig A, Goetz L, Hartmann H, Lampe D, Buchman J, et al. Direct measurement of sound velocity in various specimens of breast tissue. Invest Radiol 2000; 35:721-6.

23. Devaney. A filtered backpropagation algorithm for diffraction tomography. Ultrasonic Imaging, 4(4):336-350, October 1982.

24. S. A. Johnson and M. L. Tracy. Inverse scattering solutions by a sinc basis, multiple source, moment method. Part I: Theory, Ultrasonic Imaging, 5:361-375, 1983.

25. Natterer, F. A Propagation-Backpropagation Method for Ultrasound Tomography, Inverse Problems 11, 1225- 1232, 1995.

26. Schreiman JS, Gisvold JJ, Greenleaf JF, Bahn RC. Ultrasound transmission computed tomography of the breast. Radiology 1984; 150:523-30.

27. Claerbout, J.F. Toward a unified theory of reflector mapping. Geophysics, 36, 467-481, 1971. 


\section{Figure Captions}

Figure 1. Interaction of the US pulse with the target leads to a scattered field (left). In conventional B-scan (right) beamforming is used to launch a narrow beam into the tissue. Only the directly backscattered US energy (echo) is collected. In a tomographic approach (left) much more of the target is insonified, resulting in a scattered field that is then sampled by the detectors surrounding the target. ( $\mathrm{Tx}=$ transmitter; $\mathrm{Rx}=$ receiver). The dashed arrows represent the scattered field while the solid arrows represent the transmitted pulse (reflecting the broad beam pattern of the individual transducer elements).

Figure 2. Illustration of Snell's law showing reflection and refraction of an incident ray. Diffraction occurs when the wavelength of the US wave is comparable to the size of the scattering object. Reflection, refraction and diffraction act to alter the path of the US pulse away from a straight line trajectory.

Figure 3. Schematic of the engineering prototype scanner.

Figure 4. The experimental setup of the Karmanos scanner is shown with the cadaveric breast immersed in the water tank. The scanning process is shown in figure 5.

Figure 5: The scanning process. The receiver revolves along a circular perimeter recording signals at up to 1600 positions for each position of the transducers.

Figure 6. Cross-sections of the microcalcification phantom (a) and the cyst phantom (b). The cadaveric breast is shown in (c).

Figure 7. The geometries that define the reflected and transmitted data. Movement of the receive and transmit transducers creates effective arrays, as shown in the figure. Although the engineering prototype can synthesize a full-circular aperture any portion of the aperture can be used for image reconstruction.

Figure 8. Microcalcification phantom: (a) A schematic representation of the phantom in crosssection. The labeled arrows refer to the separations of the pairs of wires. Each wire has a radius of $0.2 \mathrm{~mm}$. (b) A B-scan image using the GE Logiq 600. The spatial resolution is approximately $1 \mathrm{~mm}$. Speckle noise and shadowing are present in the image, (c) Reflection image from the prototype scanner, showing elimination of speckle and a marked reduction in the noise level. The image is characterized by a spatial resolution of $0.4 \mathrm{~mm}$. (d) A sound speed image from the prototype scanner. The spatial resolution is approximately $1 \mathrm{~mm}$. Some artifacts are evident near the periphery of the cylinder and at the center.

Figure 9. The cyst phantom: (a) Cross-sectional schematic of the cyst phantom. The 1-cm diameter cysts are enclosed in a 4-cm diameter cylinder. (b) B-scan image made with the GE Logiq 600. The cysts are identified by the anechoic regions. The speckle noise is evident as are glint and shadowing artifacts. (c) Reflection image from the prototype scanner showing well defined cyst margins with folds and dimples of the cyst clearly delineated. These features are not seen in the B-scan image. There is no evidence of speckle but there are faint artifacts associated 
with the pulse ringing. (d) Prototype sound speed image (courtesy Techniscan Inc) also shows well defined cyst margins and good differentiation of the water, cylinder and cyst interiors.

Figure 10. The cadaveric breast. (a) An X-ray CT image showing fatty replacement and underlying fibrous architecture. (b) A single view B-scan shows only some fibrous structure along directions perpendicular to the insonifying beam (the beam illuminates the tissue downward from the top of the image). The speckle noise is evident as is the strong attenuation gradient. (c) The prototype-generated image is a superposition of refection (showing the fibrous structure) and sound speed (showing the underlying tissue and fluid) data. The resolution can only be estimated because of the absence of known structures. Analysis of the tissue fibers suggest that the resolution is $<1 \mathrm{~mm}$ and sound speed variations of $5 \mathrm{~m} / \mathrm{s}$ are discernable.

Figure 11. Reflection image of cadaveric breast (left). The corresponding X-ray CT image is shown on the right. The cadveric breast is oriented vertically (out of the page) in a cylindrical container so that the above represents a cross-sectional view.

Figure 12. Example of possible data fusion and parameter correlations. The figure on the left is an X-ray scan of the cadaveric breast used in our previous study. The slice is from a different location compared to the one shown in the earlier figure. The image on the right is an overlay of US reflectivity on sound speed (color coded: lowest sound speed = red; highest sound speed is blue). The cadaveric breast shows well-defined variations in the sound speed, ranging from 1425 $\mathrm{m} / \mathrm{s}$ to over $1500 \mathrm{~m} / \mathrm{s}$. This range of sound speed is consistent with known variations in sound speed for the breast. The lower range represents fatty tissue while the higher range represents fibrous tissue. Inspection of the figure shows that the fibrous bands are embedded within tissue that has sound speed lower than water, compatible with the fatty breast seen on the X-ray CT image. Sound speeds in the formalin medium range from 1450 to $1475 \mathrm{~m} / \mathrm{s}$, below that of water. Some enhancement of the sound speed in regions where the fibrous bands are concentrated is also evident. Such an enhancement is expected since fibrous tissue and ductal tissues have sound speed higher than fat. 

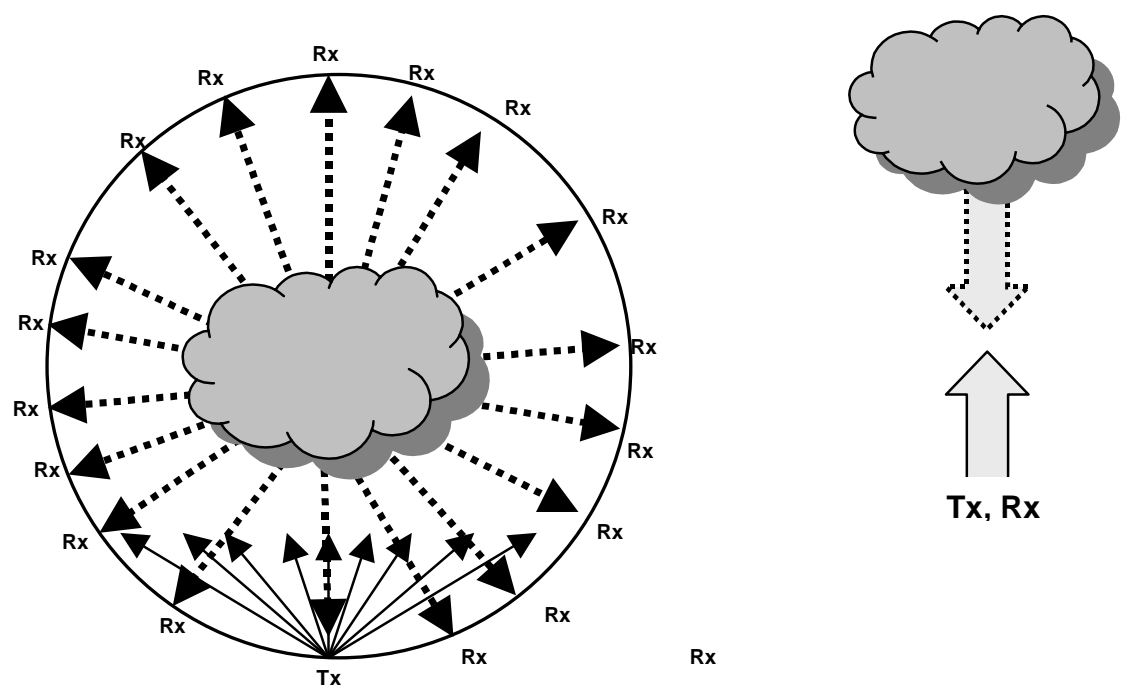

Figure 1. 

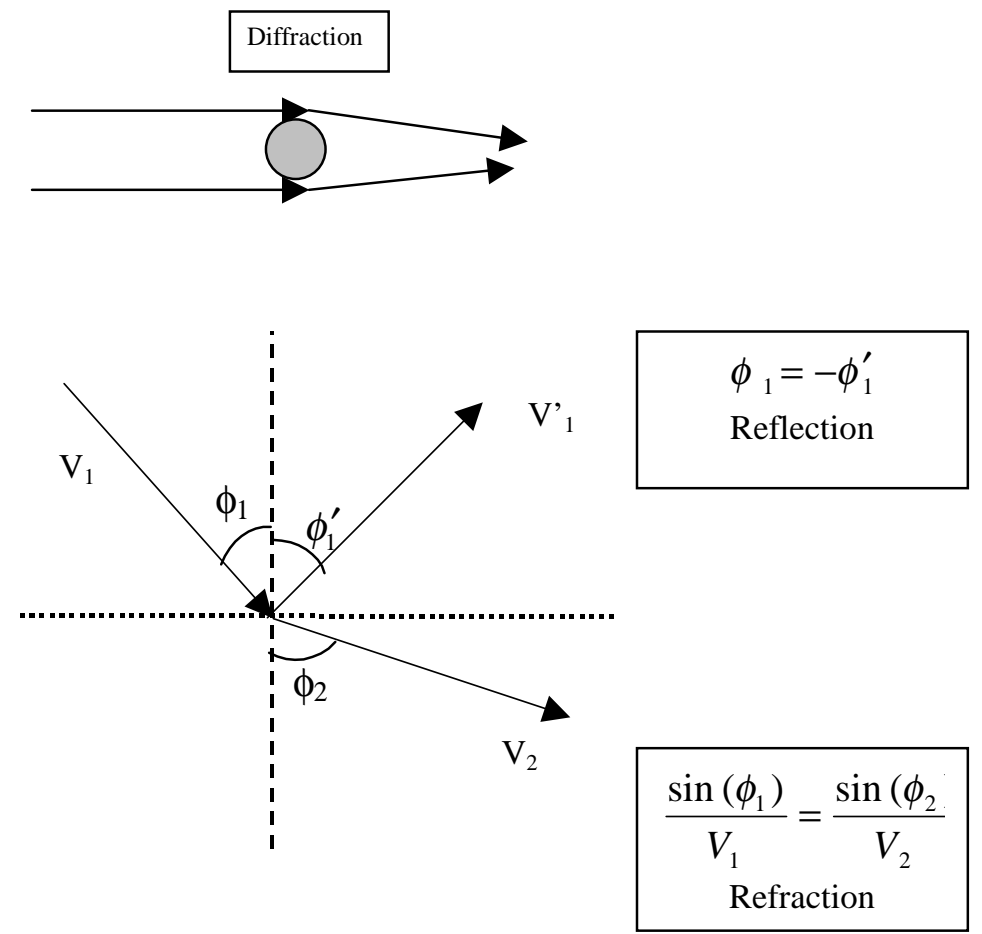

Figure 2. 


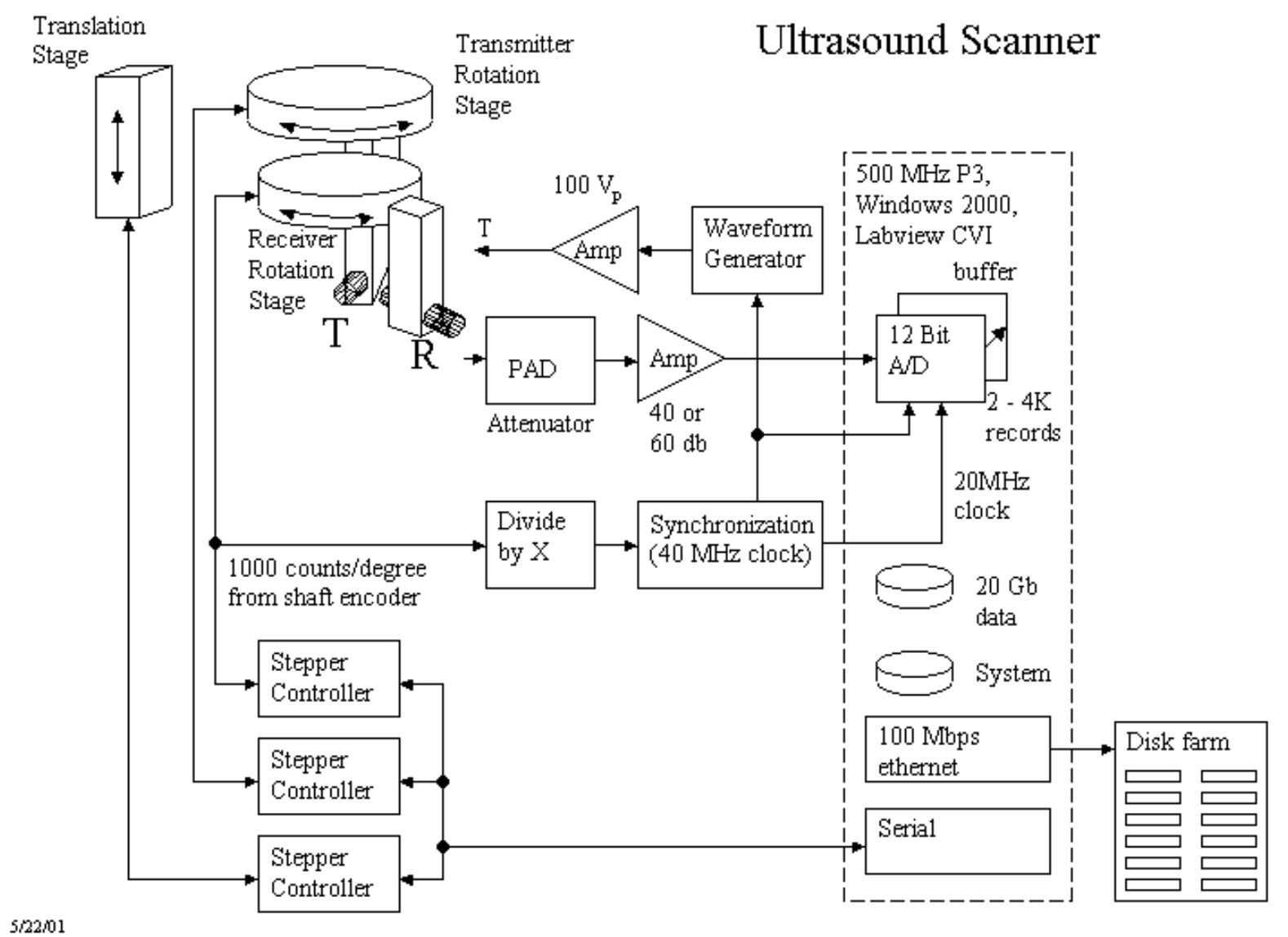

Figure 3. 


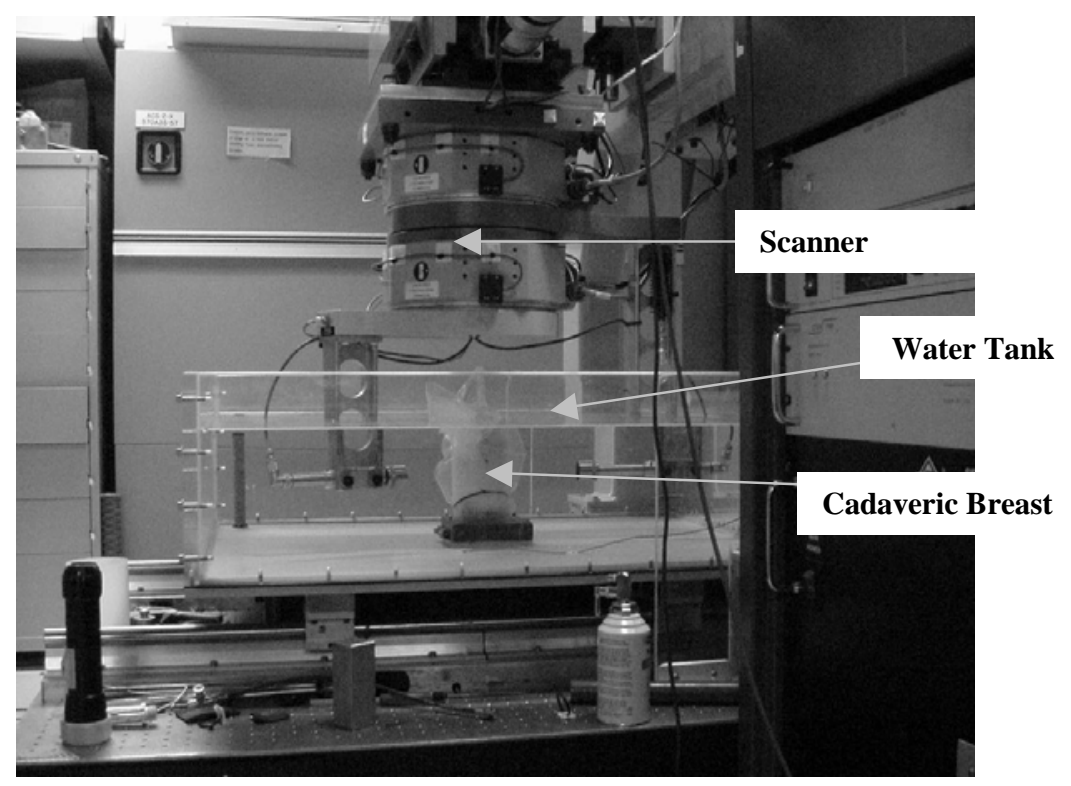

Figure 4. 


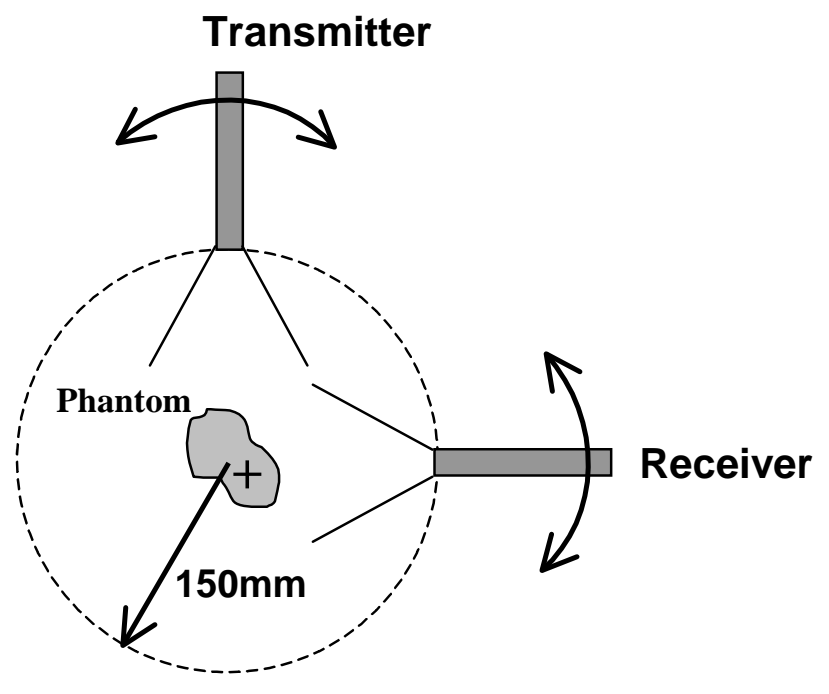

Figure 5. 


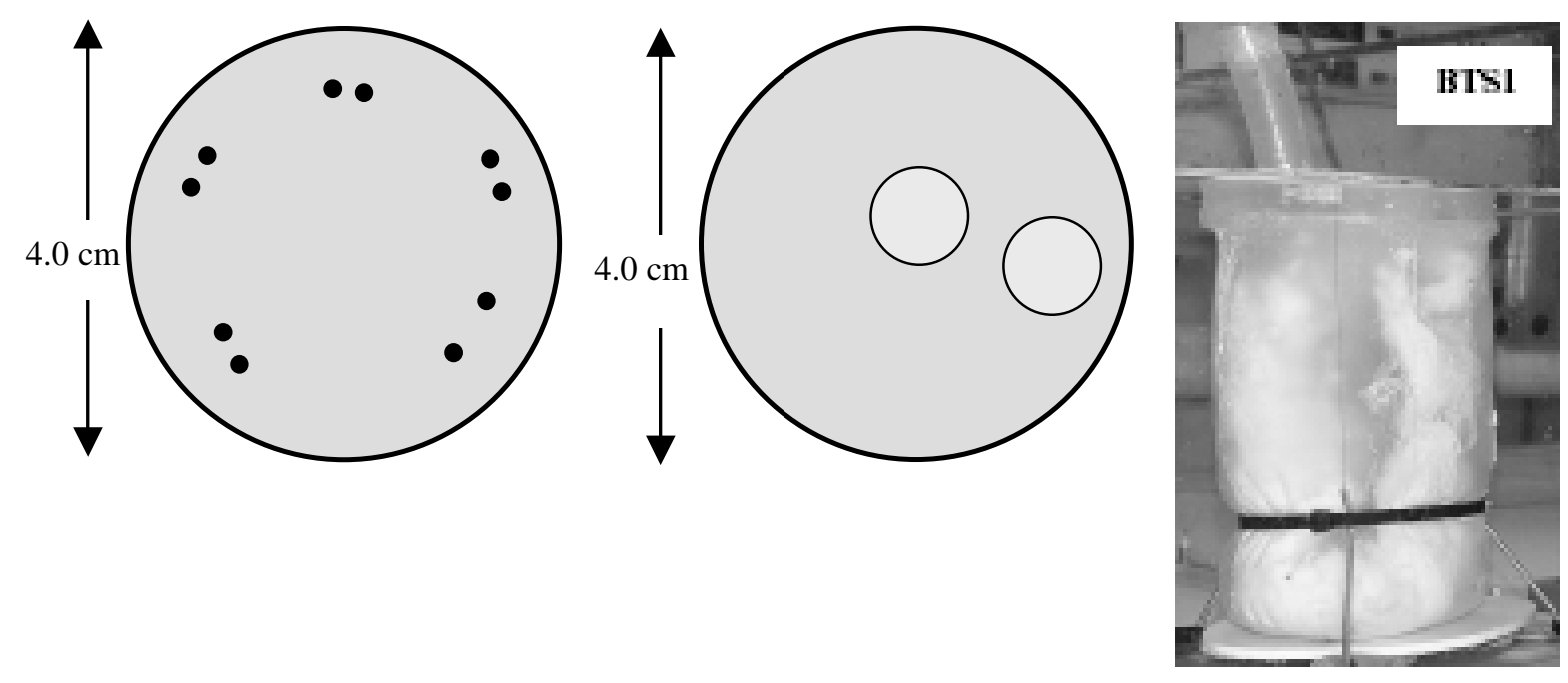

Figure 6. 


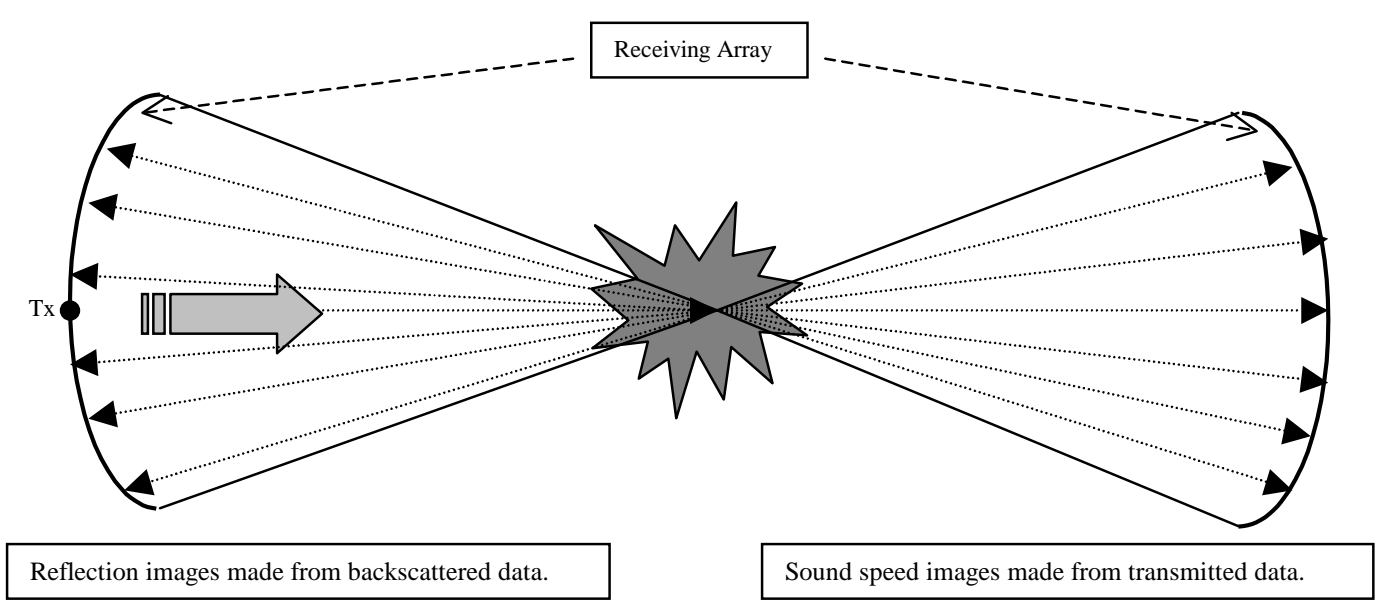

Figure 7. 
(a)

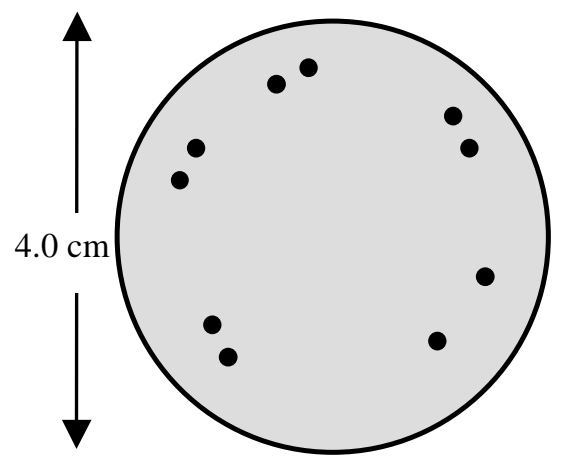

(c)

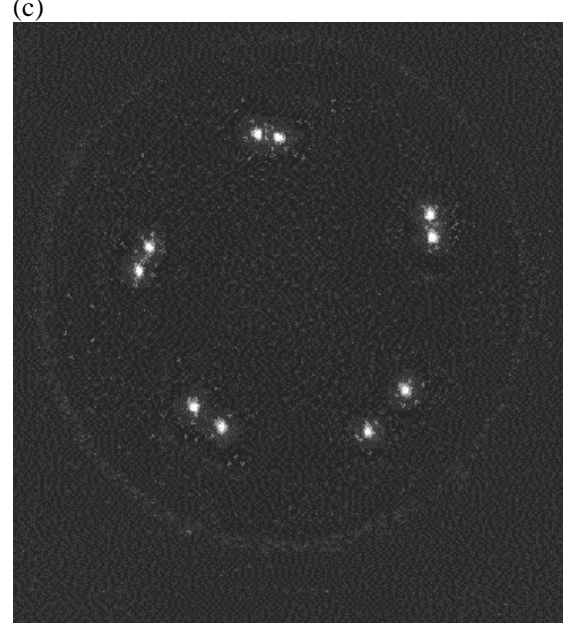

Figure 8. (b)

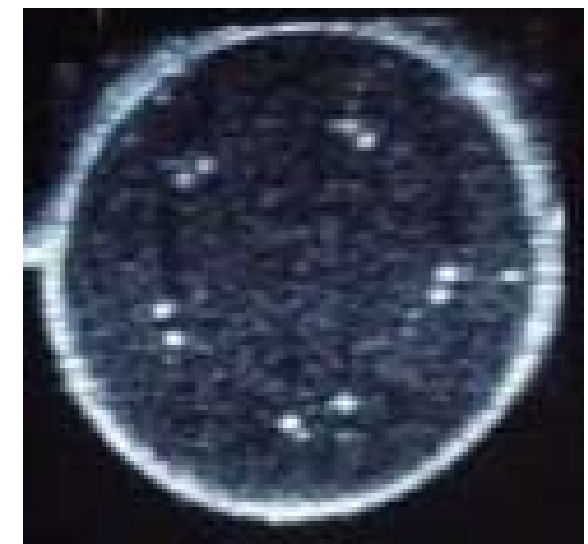

(d)

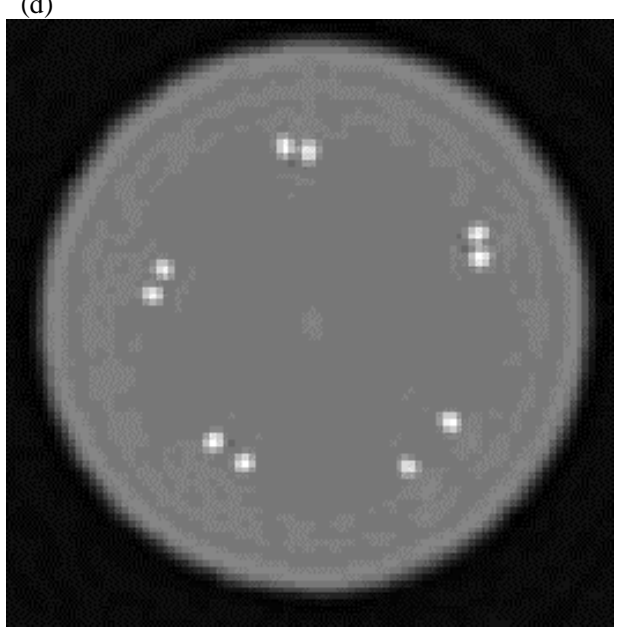


(a)

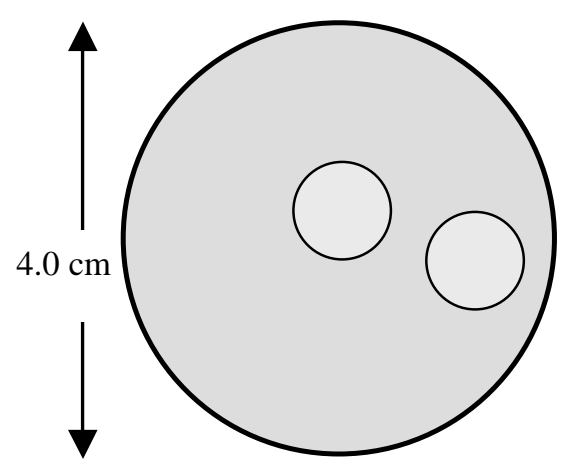

(b)

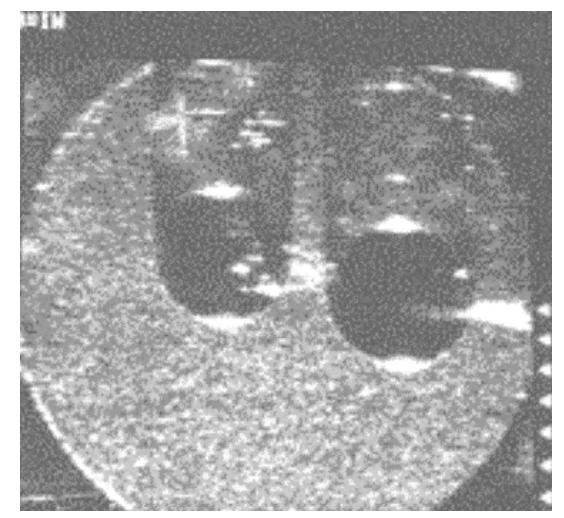

(c)

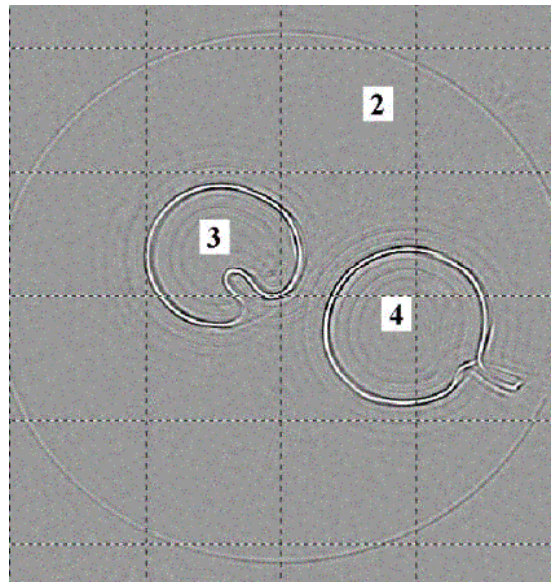

(d)

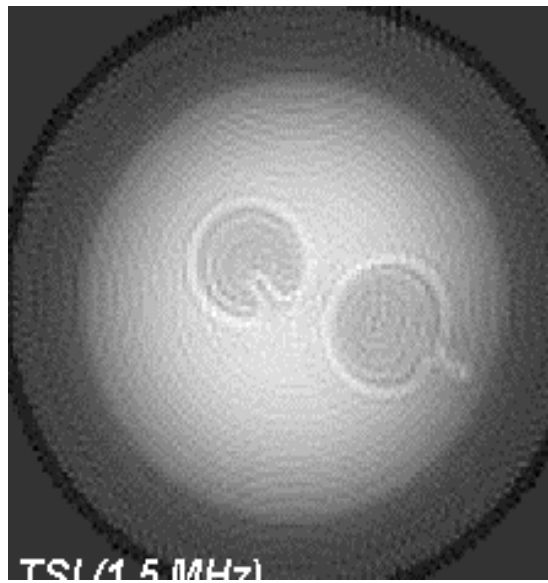

Figure 9. 
(a)

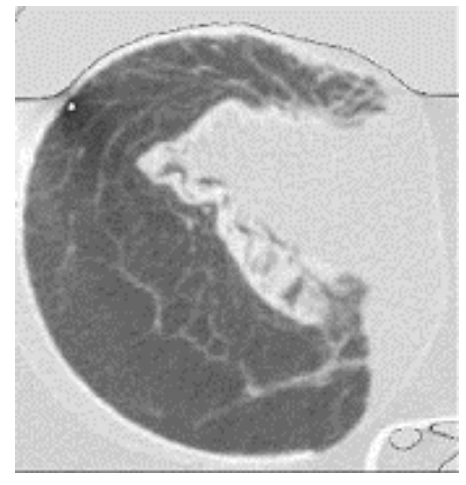

Figure 10. (b)

(c)

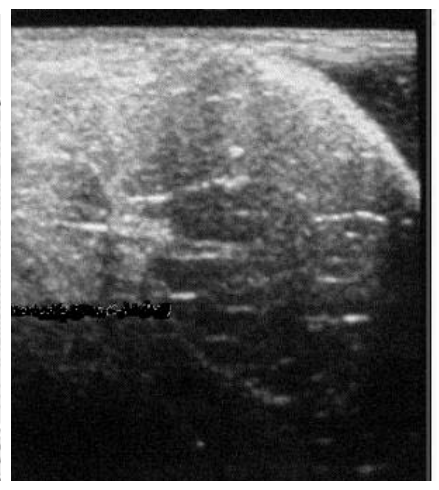

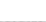

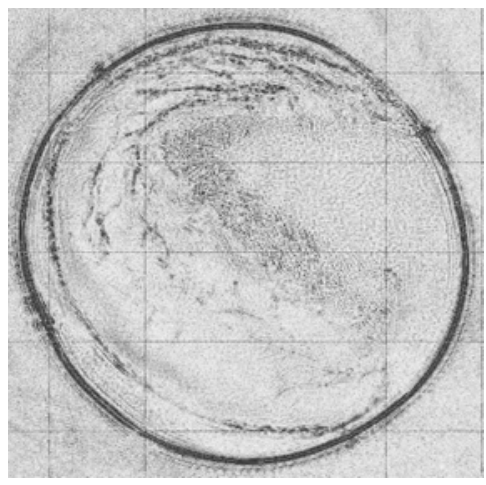



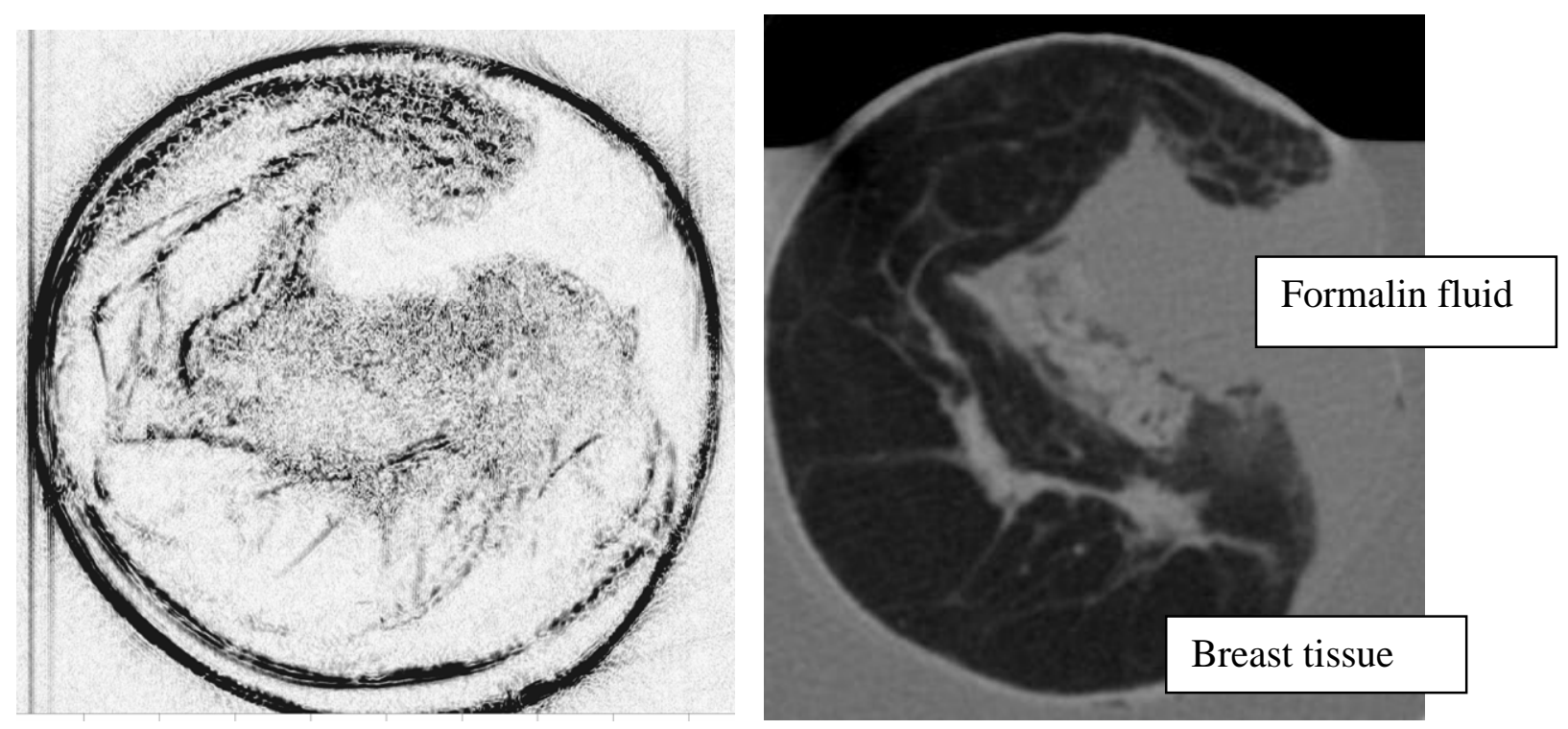

Figure 11. 

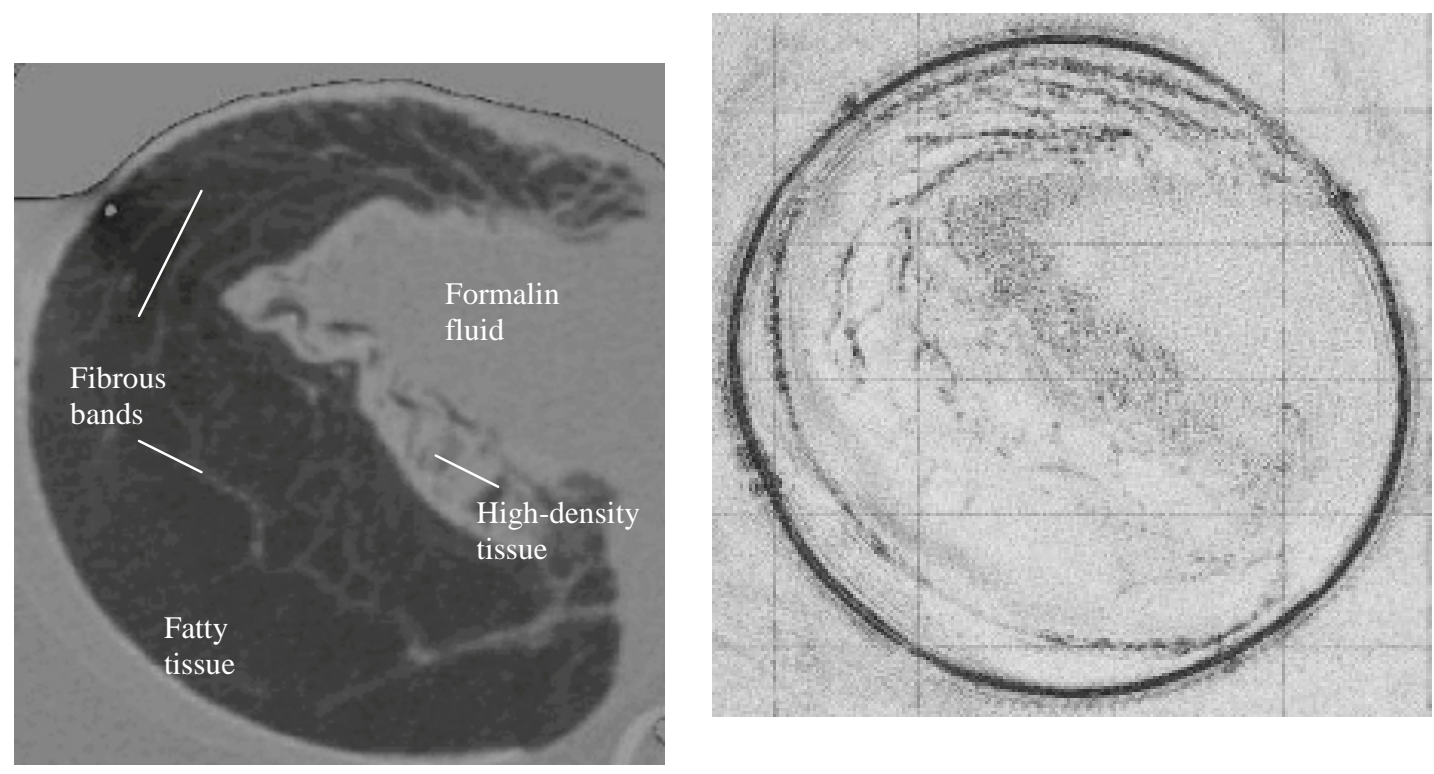

Figure 12. 